\title{
Mathematical modeling the electrical activity of the heart
}

\author{
Viacheslav V. Danilov, Roman G. Litvinov \\ Department of Applied Mathematics \\ Tomsk Polytechnic University, TPU \\ Tomsk, Russia \\ Lenin Avenue, 30
}

\author{
Olga M. Gerget \\ Department of Applied Mathematics \\ Tomsk Polytechnic University, TPU \\ Tomsk, Russia \\ Lenin Avenue, 30
}

\begin{abstract}
Nowadays, due to the prevalence of cardiovascular diseases there is extremely high demand not only in the development of new means of treatment and diagnosis, but also in their wider implementation in practice. Improving the efficiency of non-invasive diagnostic techniques, in our opinion, is one of the key tasks, the solution of which will significantly help in the treatment of cardiovascular diseases. The achievements of modern science, in particular - biology, biophysics and cybernetics, provide great opportunities to solve this problem through the development of mathematical models of cardiac electrical activity. The purpose of this article is to review the heart electrophysiology and the main approaches to the modeling of cardiac electrical activity as well as specific models.
\end{abstract}

Keywords-action potential; electrical activity of the heart; cardiac electrophysiology models; Landau-Ginzburg model; Hodgkin-Huxley model; Luo-Rudy model;

\section{INTRODUCTION}

According to the research of the World Health Organization (WHO) cardiovascular diseases (CVDs) are the number 1 cause of death globally: more people die annually from CVDs than from any other cause. It was estimated that around 17.5 million people died from CVDs in 2012, representing 31\% of all deaths in the world. Out of this number 7,4 million people died from coronary heart disease and 6.7 million died due to cerebrovascular disease (stroke). In addition, over three quarters of CVD deaths take place in low- and middle-income countries [1]. In connection with this, a variety of new methods and technologies should be introduced for timely, efficient and reliable diagnosis. Based on these facts and statistics, the main purpose of the paper is the computational modeling of the heart and some of its chambers for better understanding of its functioning as well as enabling the simulation of certain pathological conditions and medical intervention methods.

In this article, we attempted to highlight the main issues faced by all researchers, who set a goal to create a mathematical model of the heart electrical activity:

- What mechanisms make heart to work, i.e., heart electrophysiology.

- What are the different approaches of modeling the heart electrical activity, and what are their features.

\section{CARDIAC ELECTROPHYSIOLOGY}

\section{A. Electrical conduction system of the heart}

Heart muscle tissue consists of cells like any other part of the body. Cardiac conduction system controls heart contractions heart, sending electrical signals to the cells. It should be specified that the heart contains two different types of cells involved in the contractions:

- The cells of the conduction system, which pass electrical signals.

- Muscle cells, which contract heart chambers.

Each cardiac cycle is controlled and operated by electrical impulses passing through it [2]. The electric impulse (signal) propagates through the network of cells forming conductive pathways, thereby stimulating the atria and ventricles. Signal passing through the conductive pathways is possible due to a complex reaction where each cell activates the next one, thus impulse passes through the cells in a certain order. Each cell transmits electric charge sequentially, which leads to a coordinated contractions and proper heartbeat.

The electrical system causes contractions of the heart chambers and controls heart rate. It has specific conductive

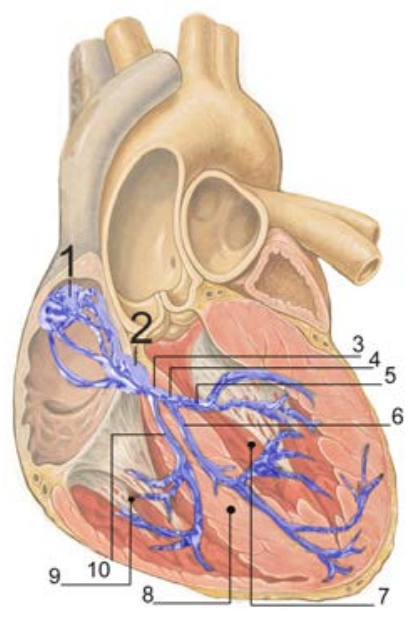

Fig. 1. Electrical conduction system of the heart. (1) Sinoatrial node; (2) Atrioventricular node; (3) Bundle of His; (4) Left bundle branch; (5) Left posterior fascicle; (6) Left-anterior fascicle; (7) Left ventricle; (8) Ventricular septum; (9) Right ventricle; (10) Right bundle branch 
pathways by which the electrical signals pass to the ventricles during each heart contraction. Excitation way of the cardiomyocytes from the sinoatrial node (SA node) to the ventricles is shown in Fig. 1.

In a healthy heart all the contractions start by the impulse formed in the sinus node (the natural pacemaker), located in the right atrium. The electrical impulse from the sinus node launches an electric chain reaction. Excitation spreads to both atria through a bundle of Bachman, thereby synchronizing their simultaneous contraction. The atria contract and pump blood into the ventricles. Then the electric excitation wave passes through a part of the heart located between the atria and ventricles and called the atrioventricular node (AV node). The electrical signal passes to the ventricles along the conductive pathways coming out of the AV node. AV node is a kind of gateway through which an electrical impulse passes from the atria to the ventricles. Pathways are in charge of delivery of the impulse to the ventricles, which are contracting, ensure the blood circulation of the body. Due to cardiac disorders there is a probability of further occurrence of additional pacemakers, which in turn entails a disturbances of the electrical activity of the heart, e.g. atrial fibrillation.

\section{B. Properties of simulated medium}

The basic subunit of the myocardium is a cardiomyocyte - a highly differentiated cell with the properties of automaticity, excitability, contractility. The structure of the cell includes a shell which is a billipid membrane. The shell has specific elements in its structure which are necessary for its functioning: membrane channels, pumps and receptors [3].

An electrical impulse propagated through the heart is called action potential. Action potential provides the property of excitability and runs each cycle of contractions. It is a short wave of depolarization during which the intracellular potential briefly becomes positive, and then returns to its initial negative level. The change of normal cardiac action potential is the timedependent variable, which is divided into several phases (see Fig. 2) [4]:

- Rapid depolarization (phase 0). This stage is characterized by the depolarization of the cell due to the opening of fast sodium channels leading to an instantaneous increase of the membrane conductance for sodium ions $\mathrm{Na}^{+}$. As a result, there is an active inflow of $\mathrm{Na}^{+}$ions, whereby the membrane potential rapidly increases to a positive value. In Fig. 1 this phase is characterized by the abrupt vertical change in the transmembrane potential.

- Early repolarization (phase 1). After the rapid depolarization, short-term recession or repolarization of the cells are observed due to fast inactivation of sodium channels and release of potassium ions from the cell.

- Plateau (phase 2). During the plateau phase there is maintained stable balance between inward movement of calcium ions $\mathrm{Ca}^{2+}$, which move into the cell through an open voltage-dependent calcium channels and outward movement of potassium ions $\mathrm{K}^{+}$, which come out of the cell through a potassium channel.
- Final rapid repolarization (phase 3). During the rapid repolarization phase the $\mathrm{Ca}^{2+}$ channels close, while the slow delayed rectifier $\mathrm{K}^{+}$channels are still open. This ensures a net outward current, corresponding to negative change in membrane potential, thus allowing more types of $\mathrm{K}+$ channel to open. This net outward, positive current (equal to loss of positive charge from the cell) causes the cell to repolarize. In Fig. 1 this is the downward slope back to the resting potential.

- Resting membrane potential or slow diastolic depolarization (phase 4). This is the resting potential and is the 'default' status of the cell, where the sodium ions, $\mathrm{Na}+$, are concentrated on the outside of the cell and the potassium ions $\mathrm{K}^{+}$are concentrated on the inside. The resting potential of a cell is negative, between $-85 \mathrm{mV}$ to $-95 \mathrm{mV}$.

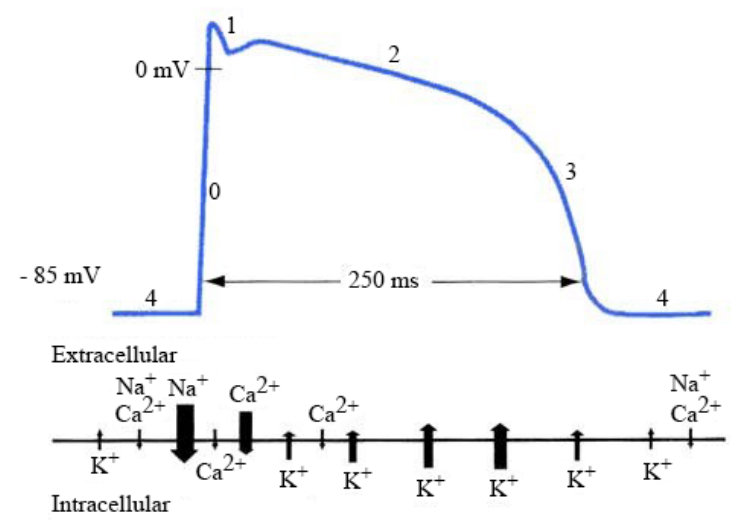

Fig. 2. The waveform and the ion currents in different phases of the action potential for ventricular cardiomyocyte. Thickness, direction and position of the arrows on the axis correspond to the intensity, direction and time of ion current.

It should be noted that action potential, described above has the "spike and dome" profile, and this is seen in the epicardial (the outer layer of heart tissue) and the $\mathrm{M}$ cell types, but not in the endocardial cells (the innermost cells). This is due to sensitivity of the transient outward current $\left(\mathrm{I}_{\text {to }}\right)$ [5]. M cells, which are also known midmyocardial or Moe cells (named in memory of Gordon K. Moe [6]), are located between the surface epicardial and endocardial layers. The main characteristics of the action potential for cardiomyocytes located in different areas of the heart are shown in the Table I. From these steps, one may conclude that for a complete modeling of the action potential of cardiac tissue it is necessary to simulate movement of various types of ions through the cell membrane and the state of different ion pumps and ion exchangers. For instance, Flavio Fenton and Elizabeth Cherry in [7] compared 45 different electrophysiological models related to the transmembrane currents and other cellular ionic processes. It is obvious that many of the approaches use electrophysiological simulations to achieve success. As an example, Richard Clayton and Alexander Panfilov in [8] consider four groups of models, in particular, the 
TABLE I. MAIN CHARACTERISTICS OF THE ACTION POTENTIAL FOR CARDIOMYOCYTES OF DIFFERENT HEART AREAS

\begin{tabular}{|c|c|c|c|c|c|}
\hline Cell type & $\begin{array}{c}\text { Diastolic potential, } \\
\mathrm{mV}\end{array}$ & $\begin{array}{l}\text { Potential change } \\
\text { rate, } \mathrm{mV} / \mathrm{ms}\end{array}$ & Overshoot, mV & Duration, ms & $\begin{array}{c}\text { Propagation speed, } \\
\mathrm{mm} / \mathrm{ms}\end{array}$ \\
\hline Sinoatrial node & $-50,-60$ & $1-10$ & 30 & $100-200$ & $0.03-0.05$ \\
\hline Atrial node & -80 & $100-200$ & 30 & $100-200$ & $0.3-0.7$ \\
\hline $\begin{array}{l}\text { Atrioventricular } \\
\text { node }\end{array}$ & $-60,-70$ & $5-15$ & 20 & $100-300$ & 0.1 \\
\hline $\begin{array}{l}\text { Bundle of His and } \\
\text { the Purkinje fibers }\end{array}$ & $-80,-90$ & 800 & 40 & $200-500$ & $2-4$ \\
\hline Ventricles & -80 & $100-200$ & 40 & $200-200$ & $0.5-0.8$ \\
\hline
\end{tabular}

simplified two-component model, the detailed biophysical models (first and second generation) and the reduced model of the heart.

\section{MODELING OF THE ELECTRICAL ACTIVITY OF THE HEART}

\section{A. Conceptual model. Landau-Ginzburg model}

Most system of the real world responds to the excitation in a predictable manner. When the increase of excitation amplitude exceeds a critical level, a stable response of the system is suddenly changed into a simple periodic oscillation. With further excitation of the system, increasing the amplitude and frequency of oscillation and waveform becomes more complex, finally turning into aperiodic chaotic motion. With the further excitation of the system, amplitude and frequency of oscillation are increased and the waveform becomes more complex, finally turning into aperiodic chaotic motion. The mechanisms that cause such oscillations are strictly nonlinear and defined for each event. Thus, the simulation should take these specific features into account. However, it is possible to simulate certain aspects of the beginning of oscillation and chaos with the help of relatively simple differential equations, such as LandauGinzburg equations.

Landau - Ginzburg equations provide a relatively simple way of modeling certain transient phenomena, reflecting a great number of dynamic systems under the influence of a strong external impact. Such phenomena can have a periodic oscillatory behavior as well as a chaotic state with gradually increasing the amplitude of oscillations and reducing their frequency.

Landau - Ginzburg equations are universal by its nature like the FitzHugh - Nagumo equations [9 - 11]. Examples of dynamic systems which can be successfully modeled using these equations are:

- Formation of vortices in the fluid cross-flow.

- Oscillatory chemical reactions such as the BelousovZhabotinsky reaction.

In our research it is possible to apply Landau - Ginzburg equation for simulation of the dynamics of spiral waves in excitable media, as well as finding the potential of the electric field generated by the heart. The equation for the function of one spatial variable with the length $L$ for the domain $\Omega=\left[-\frac{L}{2}, \frac{L}{2}\right]$ and with periodic boundary condition $u\left(-\frac{\mathrm{L}}{2}, t\right)=u\left(\frac{\mathrm{L}}{2}, t\right)$ is given by [12]:

$$
u_{t}-\left(1+i c_{1}\right) u_{x x}=u-\left(1+i c_{3}\right) u|u|^{2} \text {, }
$$

where $u_{t}$ - the first order partial derivative of $u$ with respect to $t, u_{x x}$ - the second order partial derivative of $u$ with respect to $x$ and $t, c_{1}$ and $c_{3}$ - variables reflecting the properties of the material, as well as determining the presence and nature of stable solutions.

When the condition $c_{1} \cdot c_{3}>-1$, there is only one sustainable solution:

$$
u(x, t)=e^{-i c_{3} t} .
$$

Since $|u|^{2}$ is not an analytic function of the complex variable $u$, the solution should be divided into the real and imaginary parts, $u=u_{1}+i u_{2}$. The equation should be rewrited as a system in the next view:

$$
\begin{aligned}
& u_{1 t}-u_{1 x x}+c_{1} u_{2 x x}=u_{1}-\left(u_{1}-c_{3} u_{2}\right)\left(u_{1}^{2}+u_{2}^{2}\right), \\
& u_{2 t}-c_{1} u_{1 x x}-u_{2 x x}=u_{2}-\left(c_{3} u_{1}+u_{2}\right)\left(u_{1}^{2}+u_{2}^{2}\right) .
\end{aligned}
$$

Values of potentials can be defined by the solution of the boundary-value problem for a system of nonlinear differential equations in private derivatives. Due to Ginzburg-Landau theory, equations of the distribution of electric impulses (3) and (4) have more general view:

$$
\begin{aligned}
& \frac{\partial u_{1}}{\partial t}-\Delta\left(u_{1}-c_{1} u_{2}\right)=u_{1}-\left(u_{1}-c_{3} u_{2}\right)\left(u_{1}^{2}+u_{2}^{2}\right), \\
& \frac{\partial u_{1}}{\partial t}-\Delta\left(c_{1} u_{1}+u_{2}\right)=u_{2}-\left(c_{3} u_{1}+u_{2}\right)\left(u_{1}^{2}+u_{2}^{2}\right),
\end{aligned}
$$

where $u_{1}$ - a potential of activator, $u_{2}$ - a potential of inhibitor. It is necessary to emphasize that the constants $c_{1}$ and $c_{3}$ define conductive properties of cardiomyocytes.

Boundary conditions for Ginzburg-Landau theory are isolated, thereby there is a suggestion, that no current inflows or outflows through the heart. The initial conditions ensuring a smooth transition step at $\mathrm{z}=0$, are as follows:

$$
\begin{gathered}
u_{1}(0, x, y, z)=\tanh (z), \\
u_{2}(0, x, y, z)=-\tanh (z) .
\end{gathered}
$$

\section{B. Detailed models. Hodgkin-Huxley and Luo-Rudy models}

Detailed models are used to describe the quantitative dynamics of specific systems, when requirements for the quality of modeling are high and it is necessary to compare the calculated values with the data of field experiments.

The main idea of this works was the realization of the fact that for an adequate description of action potential generation in electroexcitability cells must take into account the transmembrane and intracellular potentials and currents. Such models include the description of a large number (up to twenty) of the membrane currents, the dynamics of ions in the 
sarcoplasm, a special regulation of calcium ions, intracellular signaling elements. As a result, estimates of parameters in the model are within experimental error [13].

Hodgkin-Huxley model is the key detailed model of the formation of potential generation in electroexcitability cells, which is based on the results of experimental analysis of generation and propagation of the nerve signal in a giant (up to $1 \mathrm{~mm}$ in diameter) squid axon [14].

From an electrical point of view, Hodgkin-Huxley model is founded on the scheme, that consists of parallel-connected batteries, resistor and capacitance. The basic scheme of the model is shown in Fig. 3 [15].

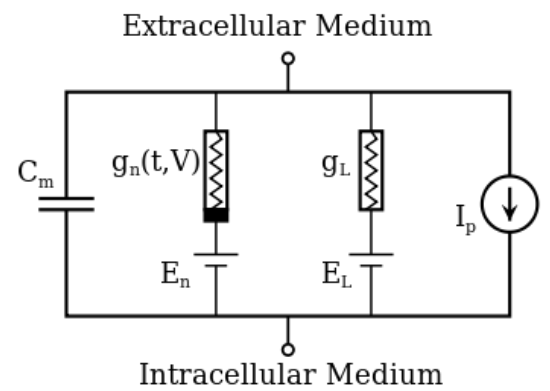

Fig. 3. Scheme of Hodgkin-Huxley model

In the scheme, each component of the excitable cell has its physical counterpart. Electrical capacitance $\left(C_{m}\right)$ corresponds to inner lipid layer of the cell membrane. Voltage-dependent ion channels are responsible for the non-linear electrical conductivity ( $G_{n}$, where the $n$ - a separate type of ion channels) - this means that conductivity is the potential time-dependent variable. This component of the system was shown by the researchers to be realized due to the protein molecules, which form voltage-dependent ion channels and each of them is marked by some probability of opening. Value of this probability depends on the electrical potential (or voltage) of the cell membrane [15].

The famous model of Hodgkin - Huxley consists of the following system of equations [16]:

$$
\begin{gathered}
C_{m} \frac{d V}{d t}+I_{\text {ion }}(V, m, h, n)=I_{s t}, \\
\frac{d m}{d t}=\frac{m_{\infty}(V)-m}{\tau_{m}(V)}=\alpha_{m}(V)(1-m)-\beta_{m}(V) m, \\
\frac{d h}{d t}=\frac{h_{\infty}(V)-h}{\tau_{h}(V)}=\alpha_{h}(V)(1-h)-\beta_{h}(V) h, \\
\frac{d n}{d t}=\frac{n_{\infty}(V)-n}{\tau_{n}(V)}=\alpha_{n}(V)(1-n)-\beta_{n}(V) n,
\end{gathered}
$$

where $I_{\text {ion }}$ is the sum of sodium, potassium and leakage currents, i.e. $I_{\text {ion }}=I_{N a}+I_{K}+I_{L}, I_{s t}-$ stimulating (external) current, $C_{m}$ - membrane capacitance, $V$ - cell potential, $\alpha$ and $\beta-$ voltage-dependent functions of gate variables, $\tau$ - time constant of gate variable, $m, n, h$ - gate variables.

An important feature of the Hodgkin-Huxley model is membrane currents approximated by smooth functions and the assumption that the channel conductivity is a function and continuously depends on the transmembrane potential. However, despite the controversy of assumptions to current data, the model still adequately describes the membrane currents because there are tens of thousands of channels, simultaneously functioning in the cell, that leads to averaging over the ensemble of channels.

Luo-Rudy model [17] created and published about forty years after the model of Hodgkin- Huxley and keeps its formalism, but takes into account a number of additional ionic currents and new formulations of dynamic changes of concentrations and flows of ions during the action potential. Creating such a complex (detailed) model became possible due to technological advance and a large number of experiments conducted in this scope of an inquiry.

The basic approach of Luo-Rudy model is based on the numerical reconstruction of the ventricular action potential with help of the formalism of Hodgkin - Huxley model. The rate of membrane potential change $\mathrm{V}$ is defined as follows:

$$
\frac{d V}{d t}=-\left(\frac{1}{C_{m}}\right)\left(I_{\text {ion }}+I_{s t}\right),
$$

where $C_{m}$ - membrane capacitance, $V$ - membrane potential, $I_{\text {ion }}$ - sum of ion currents, $I_{s t}$ - stimulating (external) current. Ionic currents are calculated as a model of ion gates, whose variables are defined as the solution of a system of nonlinear ordinary differential equations.

The format of this article does not allow us to bring all equations and experimentally calculated coefficients of LuoRudy model, so we just briefly mention features of this model. Proof of the value and versatility of the model Luo-Rudy is its wide application for simulating different parts of the heart, for example, the sinoatrial node model or with some modification (model CRN) - atrial model, while other detailed models mainly used to simulate the electrical activity of the heart ventricles.

The only weakness of this model (from a computational point of view) is the fact that the typical step for the space in the integration of Luo-Rudy model is about $0.1 \mathrm{~mm}$. It means that the modeling of only one cubic centimeter of myocardium requires at least a million cells.

\section{Results of the study}

Before turning to the direct comparison of the two approaches to modeling of the electrical activity of the heart, some features of the object of research should be noted. Firstly, the researcher always has incomplete and vague information about the object of study, in connection to this for modelling of electrical heart activity are used methods of simulation modeling, which are very complex and not always reliable (universal). Secondly, the task has complex character and can be successfully solved only in cooperation of experts in completely different areas: medicine and mathematics, physics and biophysics.

The first key difference between the conceptual and detailed models consists in goals, which should be achieved due to them. In the case of conceptual models - it is an opportunity to model heart diseases with a complex character (for example arrhythmias). In this approach, the main task is the mathematical description of the propagation of an electrical impulse in the heart in normal conditions and in case of abnormalities (disease). In the case of the detailed models - is the ability to reckon with not only a behavior of an electrical impulse in the heart, but also its nature, i.e. reasons, the conditions of its generation. In other words - detailed models 
concern the cell level (in some models - an intracellular one) allowing, for example, to understand the object of research better and to develop more effective drugs.

The second difference is in method of model development. The basis of conceptual models are equations, the laws of physics and the theory of vibrations that describe wave processes. Thus, the researcher problem reduces to the determination of physical theories, which would more fully describe the overall behavior of the electrical impulse in the heart. In developing of detailed models due to the fact that we have to take into account the cellular level, it is necessary to study and apply not only theories describing the wave processes, but also - cell theories (e.g., ion channels of cardiomyocytes), and this leads to significant complication of the model.

Accordingly, the conceptual models, as a rule, don't require a lot of computing resources. In the case of detailed models, when the model includes dozens of different types of equations, the problem becomes much more complicated - requirements to computing resources on which model is used, get higher and become a critical factor in the model efficiency.

Another important difference between models is the universality, which is caused by the factors such as availability for understanding by a wide range of people and the ability to solve a variety of problems, not only one particular. In this context, conceptual model is more attractive, because it is based on universal physical equations that make it very easy to understand: you should have a basic knowledge of biology and physics (biophysics). Detailed model requires an intimate knowledge in biology (molecular) and physics both for developing and for deep understanding, that makes the model less universal and to non-specialists on this issue. Detailed model requires both to develop and to fully understand the indepth knowledges in biology (molecular) and physics that makes it less universal and accessible to non-specialists on this issue.

\section{CONCLUSION}

In this research were analyzed and emphasized some difficulties connected with the modeling of electromechanical activity of heart. For today this scope of an inquiry is dynamically expanding and also being the pioneer, using the method of system biology, where computing and mathematical models are used for better understanding of complex biological problems.

It's important to notice that even the simulation and modeling of small amount of the heart cells are connected with a variety of problems, because the complex dynamics of big amount of ion currents, which influence the transformation of the gate variables, ion channels and membrane potential, should be considered. For today there are a multitude of published models of cardiac cells and ways of their simulation, based on experimental data. All given models is described by differential equations with a different amount of variables, starting from 2 (conceptual models) and ending with 67 (detailed models). After studying and comparing two main types of models, we can conclude that the choice of a particular model (model type) is definitely associated with the specific aim of the study and the available computing resources. In addition, some studies, such as those described in [18], show that it is possible to achieve greater efficiency of model, if researchers combine in their model strengths (simplicity) of the conceptual models, and strengths (accuracy) of detailed ones.

Unfortunately, not each model is successful and highly efficient. For example, research [19] reflects the short review of some of the most used and effective ion models for the heart ventricles.

\section{REFERENCES}

[1] Cardiovascular diseases (CVDs). Fact sheet $N^{\circ} 317$. World Health Organization. URL: http://www.who.int/mediacentre/factsheets/fs317/en

[2] R.F. Schmidt, F. Lang, G. Thews, "Physiologie des Menschen,” 29th ed., Heidelberg: Springer Medizin Verlag, 2005.

[3] S.Y. Andreev, "Modeling of atrial excitation dynamics in tasks of heart rhythm restoring,” (“Моделирование динамики возбуждения предсердий в задачах восстановления ритма сердца”) dissertation, Tomsk, 2006.

[4] R.H. Anderson, S. Yen Ho, A.E. Becker, "Anatomy and histology of the vascular system,” Heart arrhythmias, Moscow: Medicine, vol. 1, pp. 40106, 1996.

[5] I. Gussak, C. Antzelevitch, S.C. Hammill, W.K. Shen, and P. Bjerregaard, editors, "Cardiac Repolarization: Bridging Basic and Clinical Science," Humana Press, 2003.

[6] S. Sicouri, C. Antzelevitch, "A subpopulation of cells with unique electrophysiological properties in the deep subepicardium of the canine ventricle. The m cell,” Circulation Research, 1991, n. 68(6): pp. 17291741.

[7] F.H. Fenton, E.M. Cherry, "Models of cardiac cell," Scholarpedia, n. 3, 2008.

[8] R.H. Clayton, A.V. Panfilov, "A guide to modelling cardiac electrical activity in anatomically detailed ventricles," Progress in Biophysics and Molecular Biology, 2008, n. 96: pp. 19-43.

[9] R. FitzHugh, "Mathematical models of excitation and propagation in nerve,” Chapter 1 [H.P. Schwan, ed. Biological Engineering, McGrawHill Book Co., N.Y., 1969, pp. 1-85].

[10] R. FitzHugh, "Impulses and physiological states in theoretical models of nerve membrane,” Biophysical J., 1961, n. 6, pp. 445-466.

[11] R. FitzHugh, "Mathematical models of threshold phenomena in the nerve membrane,” Bull. Math. Biophysics, 1955, n. 4, pp. 257-278.

[12] V.L. Ginzburg, L.D. Landau, Zh. Eksp. Teor. Fiz. 20, 1064 (1950). English translation in: L. D. Landau, Collected papers [Oxford: Pergamon Press, 1965], p. 546.

[13] R.R. Aliev, "Computer modeling of the heart electrical activity," (“Компьютерное моделирование электрической активности сердца") Advances of Physiological Sciences, vol. 41, n. 3, pp. 44-63, 2010.

[14] A.L. Hodgkin, A.F. Huxley, "The dual effect of membrane potential on sodium conductance in the giant axon,” J Physiol, 1952. 116(4): pp. 497506.

[15] A.L. Hodgkin, A.F. Huxley, “A quantitative description of membrane current and its application to conduction and excitation in nerve," J Physiol, 1952. 117(4): pp. 500-544.

[16] J. Sundnes, G.T. Lines, X. Cai, B.F. Nielsen, K.A. Mardal, A. Tveito, "Computing the Electrical Activity in the Heart," Springer, 2006, n. 11, p. 318.

[17] C.H. Luo, Y. Rudy, "A model of the ventricular cardiac action potential, depolarization, repolarization and their interaction,” Circ. Res, vol. 68, p. 1501, 1991.

[18] E.A. Pavlov, G.V. Osipov, "Modeling of the heart activity based on mapping” ("Моделирование сердечной активности на основе отображений”), Bulletin of universities. Applied Nonlinear Dynamics, vol. 19, n. 3, pp. 104-115, 2011.

[19] P.C. Franzone, L.F. Pavarino, S. Scacchi, "Mathematical Cardiac Electrophysiology. Modelling, Simulation \& Applications,” Springer, 2014, n. 13, p. 410. 